\title{
PMSM low voltage ride through based on Coordinated Control Strategy
}

\author{
Zhang Zhaopeng ${ }^{1, *}$, Suo Dongnan ${ }^{1}$ \\ ${ }^{1}$ Datang Northeast Electric Power Test \& Research Institute, Changchun, Jilin, China
}

\begin{abstract}
Based on the analysis of the operation characteristics of the fault of direct-drive permanent magnet wind turbine and the existing protection strategies, in order to improve the low-voltage operation capability of direct-drive permanent magnet wind turbine, a new coordinated control strategy for low-voltage crossing is proposed in this paper, which includes DC brake system control of unloading circuit, double second-order generalized integral phase-locked control, extracting positive and negative sequence of grid voltage and reactive power control on grid side. Based on the common three-phase symmetrical drop fault and asymmetrical fault caused by single grounding short circuit in power grid, PASCAD simulation experiment is conducted to verify the low voltage traversing ability of wind turbine.
\end{abstract}

\section{Introduction}

At present, instantaneous voltage sag faults of wind power node account for a large proportion of wind power engineering faults ${ }^{[1]}$. If the grid voltage sag occurs and the wind generator is automatically trawled without control, it will lead to a large area of power failure and even the risk of local grid failure ${ }^{[2]}$. Therefore, countries have put forward very strict fault crossing standards. When the power grid voltage falls, the system is required to operate without going off the grid. Even the wind farm should have a certain reactive power compensation ability and be able to carry out grid-connected operation in accordance with the specified standard time requirements when the power grid fails ${ }^{[3]}$. The purpose of this paper is to make reasonable use of the advantages of permanent magnet synchronous wind turbine, and then design a low voltage crossing scheme that can ensure the continuous network operation of PMSG, which make an accurate and rapid response when the grid voltage drops. The research on PMSG low voltage crossing technology is of great significance, which can promote the large-scale development of wind power and improve the operation performance of wind power networking ${ }^{[4]}$.

\section{Control Strategy of DC Braking System Based on Unloading Circuit}

In accordance with China's low voltage traverse requirements for wind power system, it is necessary to ensure that the wind power generation system can operate on the grid for a period of time after voltage drop ${ }^{[5]}$, and the charge of the capacitor can be quickly removed.At the same time, it must control the grid-side inverter to compensate the reactive power of the system to a certain extent, and realize the low voltage through operation of the wind power generation system with the method of joint coordination control.

Fig. 1 is the control schematic diagram of the unloading protection circuit. When the system runs stably, the unloading circuit does not work; In the event of a grid failure, the power input from the rectifier side Ps is proportional to the power absorbed by the wind turbine.But the side feed into the grid inverter power will collapse because of a malfunction, resulting in a unbalanced power $\Delta \mathrm{P}$. When $\Delta \mathrm{P}$ exceeds the threshold $\Delta$ $\mathrm{Pt}, \Delta \mathrm{P}$ after PI regulator control pulse width regulator output signal.Put into the unloading circuit to release the unbalanced power of this part.A hysteresis is set in PI regulator, so they don't frequent at point $\Delta \mathrm{P}$ output signals, which reduces cutting action, reduces equipment loss and improves the system stability.

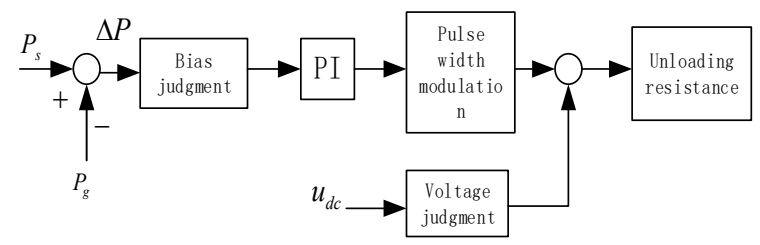

Fig.1 The schematic diagram of unloading circuit

\section{Phase lock and positive and negative sequence voltage extraction}

During normal operation, the grid-side inverter inverts the DC on both sides of the capacitor into three-phase electricity with the same amplitude and phase as the power grid.A large amount of reactive power will be generated at the grid side when the voltage of the parallel point drops instantly.At this time, in addition to putting in the unloading circuit, the grid-side inverter also needs to send

*Corresponding author’s e-mail: 1004084545@qq.com 
out a certain amount of reactive power to achieve reactive compensation $^{[6]}$. According to the requirement of low voltage crossing, the reactive power emitted by the inverter must respond quickly.In order to realize the accurate action of inverter, a phase-locked loop (PLL) technique based on double order generalized integral is proposed to detect the voltage in parallel nodes.

The phase-locked loop (PLL) based on double-second order generalized integral is used to realize the phaselocked system of the grid-connected wind power inverter system, and the positive and negative sequence components of the asymmetric grid voltage can be detected.The transient symmetry component of the flockin loop is obtained by an adaptive filter based on a secondorder generalized integrator. According to the following variation formula (1), the asymmetric voltage vector $\boldsymbol{u}_{a b c}$ is decomposed into instantaneous positive, negative and zero sequence components. The decomposition formula is shown in (2), (3) and (4) :

$$
\begin{gathered}
u_{a b c}=u_{a b c}^{+}+u_{a b c}^{-}+u_{a b c}^{0} \\
u_{a b c}^{+}=\left[T_{+}\right] u_{a b c} ;\left[\begin{array}{l}
u_{a}^{+} \\
u_{b}^{+} \\
u_{c}^{+}
\end{array}\right]=\frac{1}{3}\left[\begin{array}{ccc}
1 & a & a^{2} \\
a^{2} & 1 & a \\
a & a^{2} & 1
\end{array}\right]\left[\begin{array}{l}
u_{a} \\
u_{b} \\
u_{c}
\end{array}\right] \\
u_{a b c}^{-}=\left[T_{-}\right] u_{a b c} ;\left[\begin{array}{l}
u_{a}^{-} \\
u_{b}^{-} \\
u_{c}^{-}
\end{array}\right]=\frac{1}{3}\left[\begin{array}{ccc}
1 & a^{2} & a \\
a & 1 & a^{2} \\
a^{2} & a & 1
\end{array}\right]\left[\begin{array}{l}
u_{a} \\
u_{b} \\
u_{c}
\end{array}\right] \\
u_{a b c}^{0}=\left[\begin{array}{l}
\boldsymbol{T}_{0}^{0} \\
u_{a} \\
u_{b}^{0} \\
u_{c}^{0}
\end{array}\right]=\frac{1}{3}\left[\begin{array}{lll}
1 & 1 & 1 \\
1 & 1 & 1 \\
1 & 1 & 1
\end{array}\right]\left[\begin{array}{l}
u_{a} \\
u_{b} \\
u_{c}
\end{array}\right]
\end{gathered}
$$

$T$ is the matrix transformation, a is the phase shift of the fundamental wave frequency added to the instantaneous sinusoidal input signal, which is equivalent to $120^{\circ}$ phase shift, and is a special form of Fortescue operator. The research of controlling three-phase gridconnected converters mainly focuses on the positive sequence and negative sequence components of voltage.Each order component in $u_{a b c}$ is transformed into (6) by transformation matrix (5).

$$
\begin{aligned}
T_{a b c / \alpha \beta}=\frac{2}{3}\left[\begin{array}{ccc} 
& -\frac{1}{2} & -\frac{1}{2} \\
0 & \frac{\sqrt{3}}{2} & -\frac{\sqrt{3}}{2}
\end{array}\right] \\
u_{\alpha \beta}^{+}=\left[T_{\alpha \beta}\right] u_{a b c}^{+} \\
u_{\alpha \beta}^{-}=\left[T_{\alpha \beta}\right] u_{a b c}^{-}
\end{aligned}
$$

$u_{\alpha \beta}^{+}$is the positive sequence component of voltage, and $u_{\alpha \beta}^{-}$is the negative sequence component of voltage.Finally, we can compute these matrices to obtain the following expression:

$$
\begin{gathered}
u_{\alpha \beta}^{+}=\left[T_{\alpha \beta^{+1}}\right] u_{\alpha \beta} ;\left[T_{\alpha \beta^{+1}}\right]=\frac{1}{2}\left[\begin{array}{cc}
1 & -q \\
q & 1
\end{array}\right] \\
u_{\alpha \beta}^{-}=\left[T_{\alpha \beta^{-1}}\right] u_{\alpha \beta} ;\left[T_{\alpha \beta^{-1}}\right]=\frac{1}{2}\left[\begin{array}{cc}
1 & q \\
-q & 1
\end{array}\right] \\
q\left(q=e^{\frac{-j w}{2}}\right) \\
\text { represents the phase shift operation of lag }
\end{gathered}
$$

$90^{\circ}$, which can obtain the quadrature axis component of the input waveform when applied to the time domain.

$u_{\alpha}^{\prime} 、 u_{\beta}^{\prime} 、 q u_{\alpha}^{\prime}$ 和 $q u_{\beta}^{\prime}$ is the direct and quadrature axis signal in the $\alpha$ and $\beta$ components of the input vector. They are generated by two second order generalized integrators - orthogonal signal generators. These signals serve as input signals to the Positive/Negative Sequence Component Computing Module (PNSC). The formulas for each order component on axis $\alpha \beta$ are (7) and (8). The positive and negative sequence components of the fundamental frequency are obtained by decoupling PNSC, and the phase and frequency of the positive sequence components of the fundamental frequency are detected by phase-locked loop.Finally, the asymmetric input voltage vector is transferred to the positive sequence component by a biquadratic generalized integrator.The function formula (9) is as follows:

$$
u_{\alpha \beta}^{+}=\left[T_{\alpha \beta^{+}}\right] u_{\alpha \beta}=\frac{1}{2} \frac{k \omega}{S^{2}+k \omega s+\omega^{2}}\left[\begin{array}{cc}
S & -\omega^{\prime} \\
\omega & S
\end{array}\right] \omega
$$

The structure diagram of the three-phase phase-locked loop of the two-order generalized integrator is shown in Figure 2:

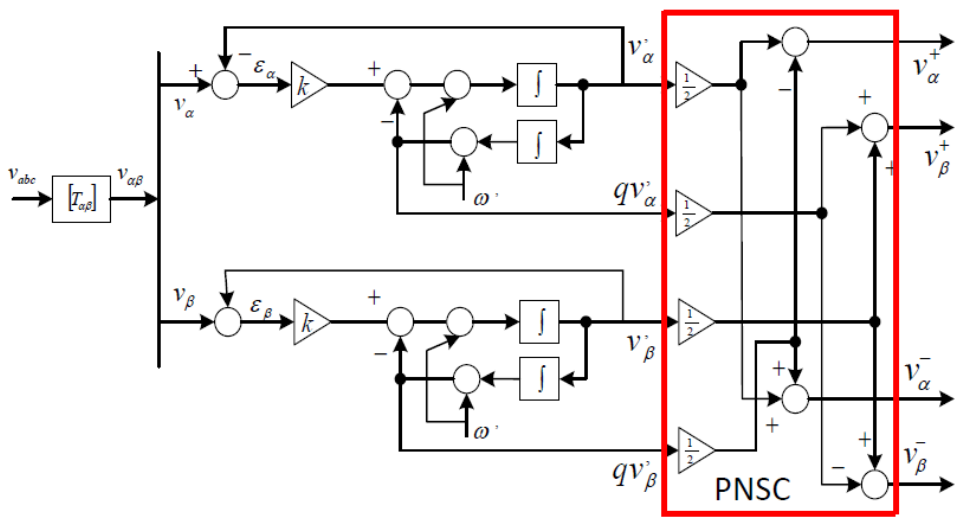

Fig.2 Structure diagram of bi-second-order generalized integral phase-locked loop 
In this paper, the frequency locked loop is used instead of the phase locked loop, and the signal $U \alpha$ and $U \beta$ as the input of the frequency locked loop system of the biorder generalized integrator have the same frequency.The frequency difference signals generated by $\alpha$ and $\beta$ signal generators are combined using the method of calculating the mean error signal:

$$
\varepsilon_{f}=\frac{\varepsilon_{f(\alpha)}+\varepsilon_{f(\beta)}}{2}=\frac{\varepsilon_{\alpha} q u_{\alpha}^{\prime}+\varepsilon_{\beta} q u_{\beta}^{\prime}}{2}
$$

$\varepsilon_{f}$ is the combined frequency difference signal. $\varepsilon_{f(\alpha)}$ and $\boldsymbol{\varepsilon}_{f(\beta)}$ are $\alpha$ and $\beta$ frequency difference signals respectively. The two order generalized integrator frequency-locked loop realizes the decoupling estimation of the symmetric components of the three-phase voltages and the estimation of the grid frequency at the $\alpha$ and $\beta$ reference coordinates. The detection and control algorithm can realize the fast and accurate detection of grid faults, especially the asymmetric faults on the grid side, which judge the faults in the first time.

\section{Reactive power compensation control of grid side converter}

When grid voltage sag occurs, grid side will produce a lot of reactive power demand.The directional $\mathrm{dq}$ axis decoupling control is adopted for the PWM converter on the grid side.Axis $\mathrm{d}$ and Axis q control DC voltage and reactive current respectively, so the reactive power compensation of grid voltage can be realized by adjusting the current of Axis q.

When the grid-side voltage drops, the grid-side converter is in the state of reactive compensation. At this time, the current reference value of dq axis is reset, and the active current $i_{\text {reactive }}$ for reactive compensation is calculated through the PI regulator of the outer loop of the grid voltage. With the help of formula (11), the maximum active current $i_{\text {active }}$ provided by the outer voltage loop in reactive power compensation is calculated, and the value of $i_{\text {active }}$ and the reference value of the original active current $i_{\text {gdref }}$ is logically judged. If $i_{\text {active }}$ is large, it means that the reactive power compensation capacity of the outer voltage loop is enough to stabilize the DC voltage and ensure the stability of the system.If $\dot{I}_{\text {active }}$ is small, it means that the reactive power compensation ability of the outer voltage loop cannot stabilize the DC voltage.In order to ensure the stability of the system, it is necessary to input the unloading circuit through the PI regulator to consume the excess reactive power.

$$
i_{\text {active }}=\sqrt{i_{\max }^{2}-i_{\text {reactive }}^{2}}
$$

$i_{\max }$ is the maximum current value that the grid side converter is allowed to pass through in the formula.

The positive sequence voltage of the grid side is extracted and compared with $90 \%$ of the rated voltage. When the grid side converter detects that the positive sequence voltage of the grid is less than $90 \%$ of the rated voltage, it delays $30 \mathrm{~ms}$ to enter the low voltage traverse.During low voltage crossing, reactive current takes precedence over control.The grid side converter sends reactive current according to the depth of voltage sag to support grid voltage recovery.After the grid voltage is restored, the converter exits the low-voltage through-through operation mode with a delay of $20 \mathrm{~ms}$.

The reactive current control mode during low voltage traverse is as follows:

$$
i_{\text {reactive }}=2\left(0.9-U_{\text {positive }}\right) I_{N}
$$

$I_{\mathrm{N}}$ is the rated current of the wind farm.

In case of symmetrical sag of grid voltage, the fluctuation range of reactive current during low voltage crossing is: [0-50\%]; In the case of asymmetric sag of grid voltage, the range of reactive current fluctuation during low voltage crossing is [0-40\%].Through the above analysis, the control strategy of the network side converter is improved.It can provide reactive power compensation and stabilize the power supply voltage in time when the measuring voltage falls down.The control schematic diagram of the improved grid-side converter is shown in Figure 3.

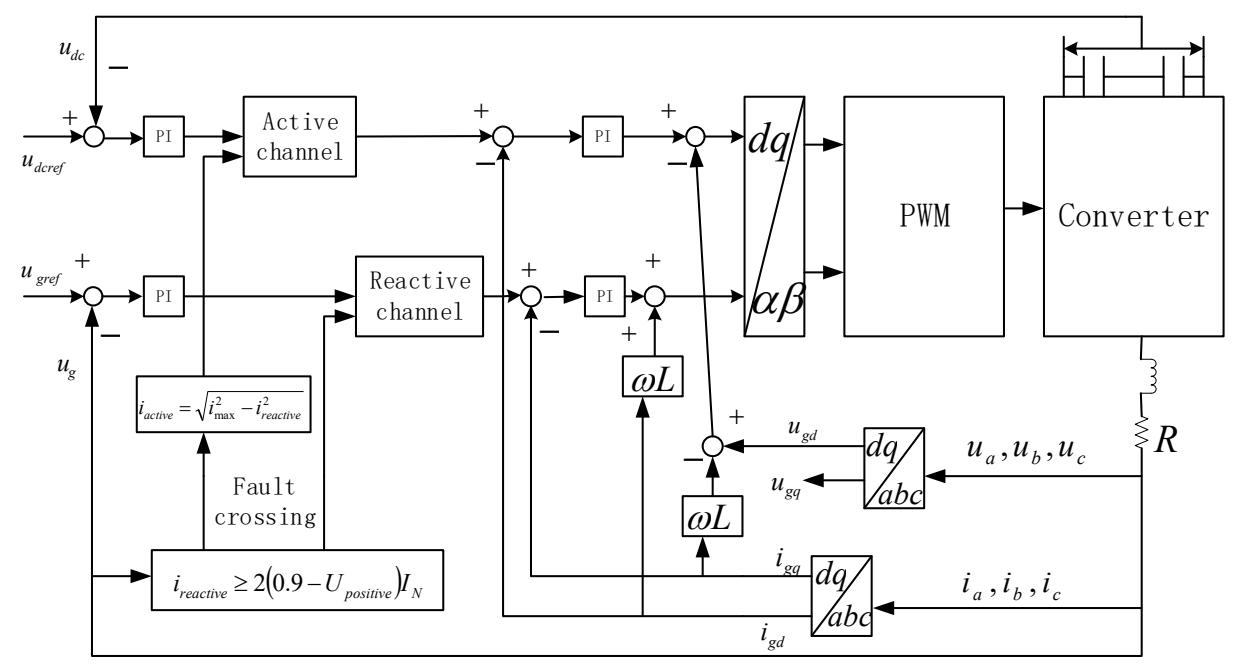

Fig.3 Control schematic diagram of grid-side converter 


\section{Simulation analysis of optimized low voltage traverse control strategy}

Fault condition: the direct drive permanent magnet wind power generation system runs at the experimental wind speed $(9.3 \mathrm{~m} / \mathrm{s})$ and the voltage at the middle side of the grid runs at $28.5 \mathrm{kV}$. The three-phase symmetrical voltage drop fault occurs in the grid at $1.5 \mathrm{~s}$. The failure duration was $1.85 \mathrm{~s}$ and the drop depth was $50 \%$. The simulation graph of the control theory adopted in this paper is as follows.

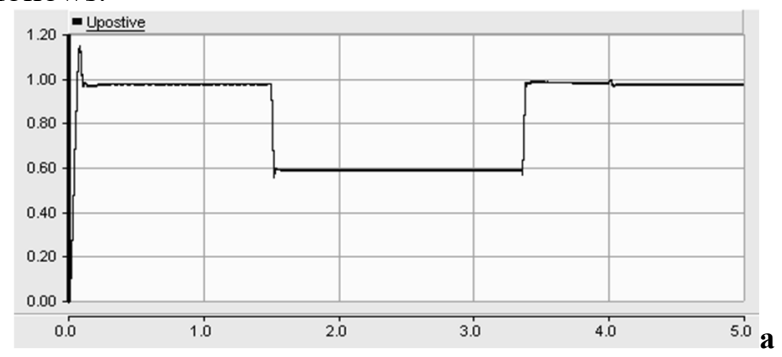

Grid positive sequence voltage per unit value
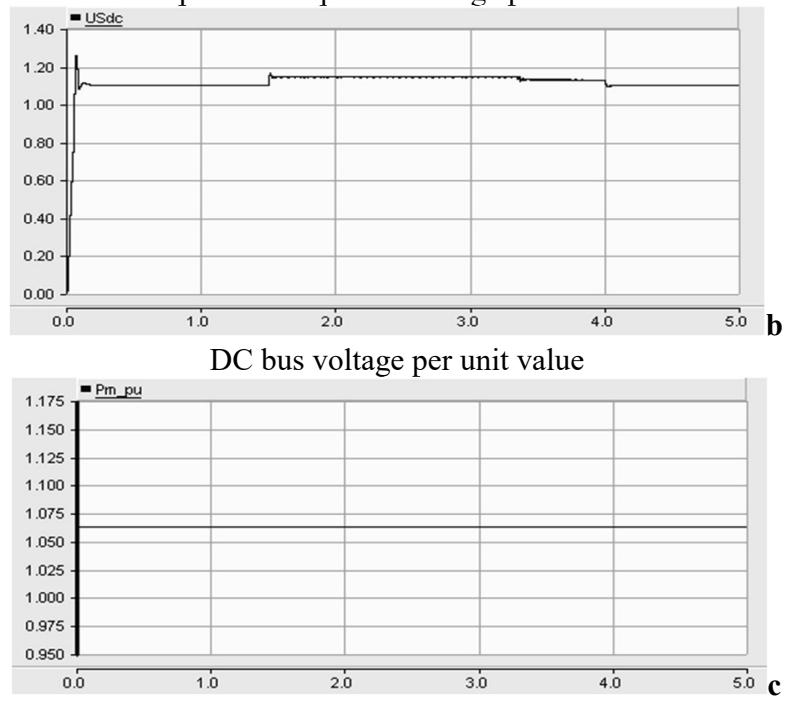

Machine side output active power per unit value

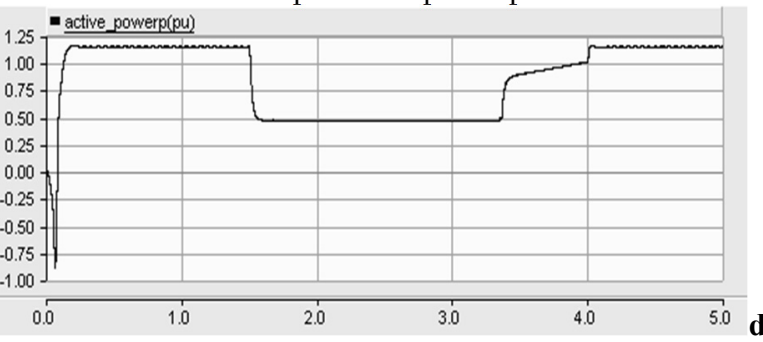

Grid side output active power per unit value

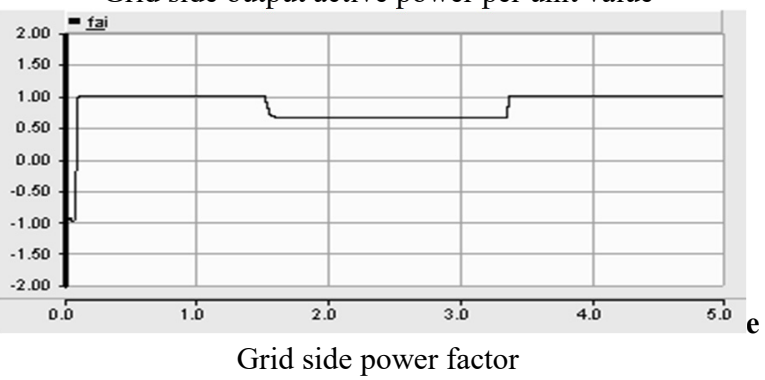

Grid side power factor

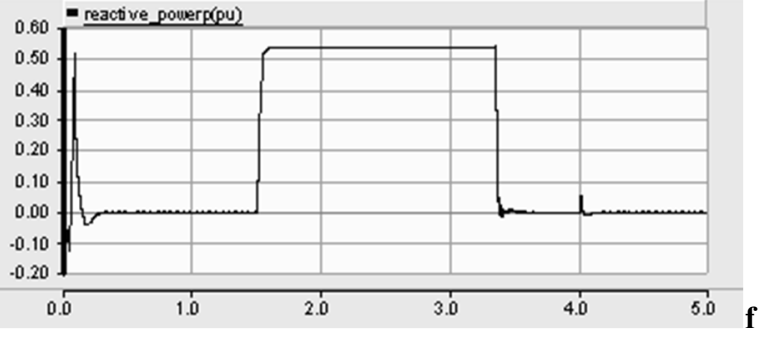

Grid side output reactive power per unit value

Fig.4 The simulation waveform of the improved control strategy when the voltage drops by $50 \%$.

After the improved control strategy, the grid side active power decreases by about $58.26 \%$, the reactive power compensation decreases by about $53.39 \%$, and the power factor decreases by about 33\% during the same grid fault period.The active and reactive power parameters have reached the standard. Although some power factor is lost, it can ensure that the fan does not run off the grid during the power grid failure, and guarantee the high quality power output.

\section{Conclusion}

In this paper, the mathematical model and operation control method of direct drive permanent magnet synchronous wind turbine are studied.Based on the analysis of the reasons of DC capacitive voltage fluctuation caused by the power imbalance on both sides of the power network and the conventional DC braking control strategy, the advantages and disadvantages of the conventional control strategy of the direct-drive permanent magnet wind turbine in the process of low voltage crossing are summarized.This paper selects a new coordinated control strategy for low voltage traversing, which includes unload resistance control, phase-locked control, positive and negative voltage sequence extraction and grid side reactive power control.This strategy improves the voltage stability of the power grid, realizes the safe crossing of the wind turbine during the period of low voltage failure, and improves the stability and practicability of the system.By building a simulation model, the conventional control method is not in line with the actual requirements.It realizes the problem of low penetration but can not be connected to the grid, which verifies the effectiveness and superiority of the new LVRT control strategy.

\section{References}

1. CONG Cong Active control of rotational blade in grid-connected wind turbine with tuned mass damper[J]. Renewable Energy Resources, 2019, 37(2): 267-271.

2. TIAN Jun, ZHANG Wenyi, WANG Yupeng, et al. Modeling and Analysis of Sub-Synchronous Oscillation in Wind Power Grid Connection System[J], Distributed Energy, 2018, 5(3): 23-27.

3. Alepuz S, Alejandro C, Sergio B M, et al. Use of Stored Energy in PMSG Rotor Inertia for Low- 
Voltage Ride-Through in Back-to-Back NPC Converter-Based Wind Power Systems[J]. IEEE Transactions on Industrial Electronics, 2013, 60(5): 1787-1796.

4. Kim K G, Jeung Y C, Lee D C, Kim H J. LVRT Scheme of PMSG Wind Power Systems Based on Feedback Linearization[J]. IEEE Transactions on Power Electronics, 2012, 5(27): 2376-2384.

5. ZHANG Wentao, WANG Xiuli, LI Yan, et al. An Analysis Model of Multi-Area Interconnected Power Systems With Large-Scale Wind Power Involved in Comprehensive Heating and Power System Scheduling[J]. Power System Technology, 2018,42(1): 154-161.

6. Wang Xiaoyu. Research on Technologies of Low Voltage Ride Through for Grid-connected Directdriven Permanent Magnet Wind Power Systems Beijing: North China Electric Power University, 2018. 